\title{
Grohn's disease: A case report with literature review
}

\author{
${ }^{1}$ Clinical Service of Pathology, Emergency County Hospital, Constanta \\ ${ }^{2}$.Department of Pathology, Faculty of Medicine, „Ovidius” University, Constanta
}

\begin{abstract}
Crohn's disease is an idiopathic inflammatory disorder which can affect any segment of the digestive tract. Generally considered uncommon and often underestimated, it can endanger the patient's life due to its local and systemic complications. In this article we present the case of a 67-year-old male patient who was admitted for cramping abdominal pain, nonbloody diarrhea, fever and anorexia. He described a 5-year history of similar episodes composed of the same symptoms for which he was admitted. In the past no diagnosis was confirmed and he received no treatment, due to the fact that the episodes were autolimited and the patient didn't ask for medical attention. In this case surgery was required and the diagnosis of Crohn's disease was histopathologically confirmed, thus leading to a proper choice of treatment to avoid possible complications.
\end{abstract}

Keywords: Chron's disease, enterocolitis, digestive symptoms

Mariana Aschie

Department of Pathology, Faculty of Medicine, "University "Ovidius" Constanța

Clinical Service of Pathology, Emergency County Hospital

145 Tomis Avenue, 900591 Constanţa, Romania

$+40745043505$

aschiemariana@yahoo.com

\section{Introduction}

Crohn's disease, also referred to as regional enteritis, granulomatous enterocolitis, and terminal ileitis, is a chronic relapsing and remitting inflammatory disease of unknown cause that is often multifocal and can affect any portion of the gastrointestinal tract [1]. It is generally accepted that the Scottish surgeon Dalziel gave the first account of the disease in 1913 [2].In the 1960s Lockhart-Mummery and Morson described the involvement of the large intestine by Crohn's disease [3] and it was in the 1950s that anal and anorectal Crohn's disease were described [4]. In time it became clear that Crohn's disease can involve any part of the digestive tract and that extraintestinal manifestations can be present, especially in the skin, eyes and joints [5-9]. We discuss, through the use of a case report, the histomorphologic features of Crohn's disease involving both the small and large intestine in a patient. 


\section{Case report}

A 67-year-old man was admitted with a 36-h history of cramping abdominal pain, mainly localized to the left lower quadrant of the abdomen, diarrhea, fever and anorexia. He described a 5-year history of cramping abdominal pain associating nonbloody diarrhea and occasionally fever. In the past no diagnosis was confirmed and he received no treatment, due to the fact that the episodes were auto-limited and the patient didn't ask for medical attention. On retrospective questioning he denied any extraintestinal manifestations and familial association. Blood investigations revealed ared cell count of $3.69 \times 10^{12} / \mathrm{L}$, a white cell count of $9.11 \times 10^{9} / \mathrm{L}$ and a C-reactive protein of $39.6 \mathrm{mg} / \mathrm{L}$. Due to the acute state of the patient and because Crohn's disease was suspected, endoscopy was not performed, thus avoiding the risk of iatrogenic bowel perforation. Following a 4-day observation under curative treatment, the patient's state imposed surgery, ileocolectomy and segmental enterectomy being performed.

Gross findings included a variable circumference for both the ileum and colon, with dilated areas alternating with normal or narrow areas, flattened folds of the ileum's mucosa with a thin, friable ileal wall and an irregular pseudopolypoid appearance of the colonic mucosa (Figure 1) with multiple longitudinal "rake" ulcers. Crohn's disease was histopathologically confirmed.

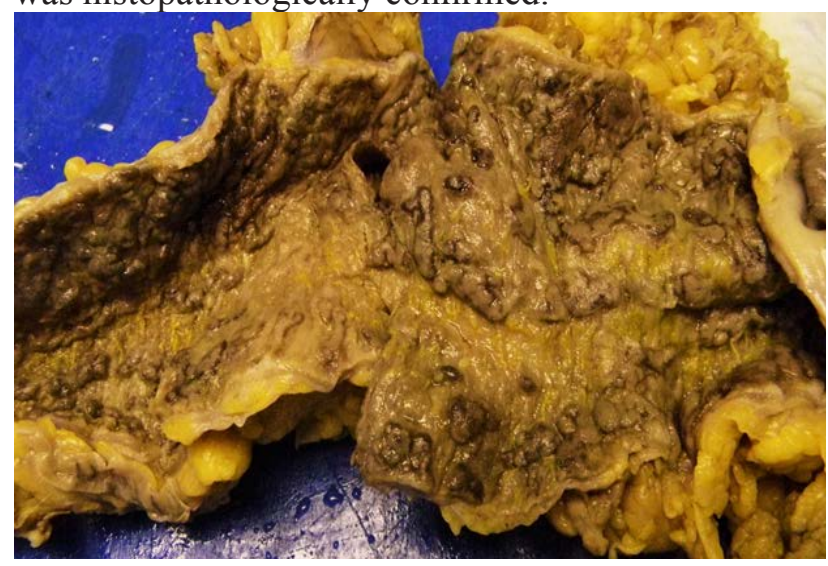

Figure 1 - Gross picture of the specimen-Colonic mucosa.
A multifocal inflammatory process was found, with areas of submucosal fibrosis and transmural inflammation. Lymphoid aggregates were described in the submucosa, some of them tending to organize in granulomas (Figure 2A-C). Ulcers were found, most of them longitudinally oriented, involving the mucosa, submucosa and breaching the muscularis propria (Figure 3A-C); in some areas they also involved the serosa (Figure 4). Overall the histological features were conclusive for Crohn's disease in both the small and great intestine.
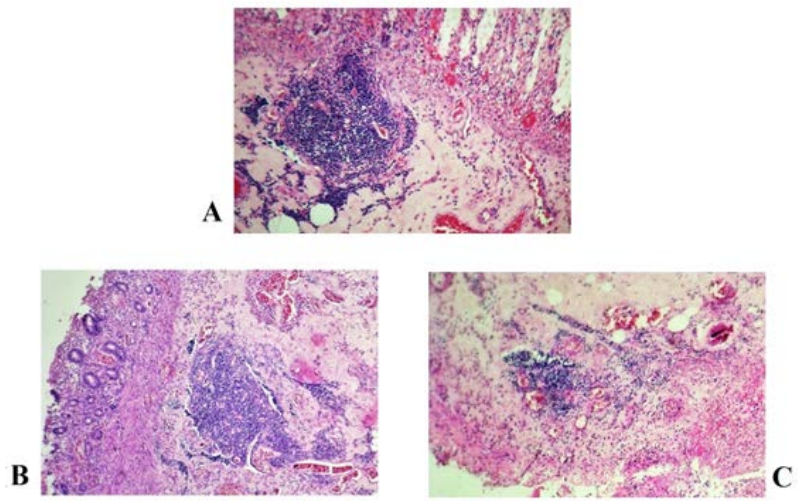

Figure 2 - Lymphoid aggregates in the submucosa, some of them tending to organize in granulomas $[(A) H \& E$ stain $40 \times$; (B) $H \& E$ stain $4 \times$; (C) $H \& E$ stain $10 \times]$

A
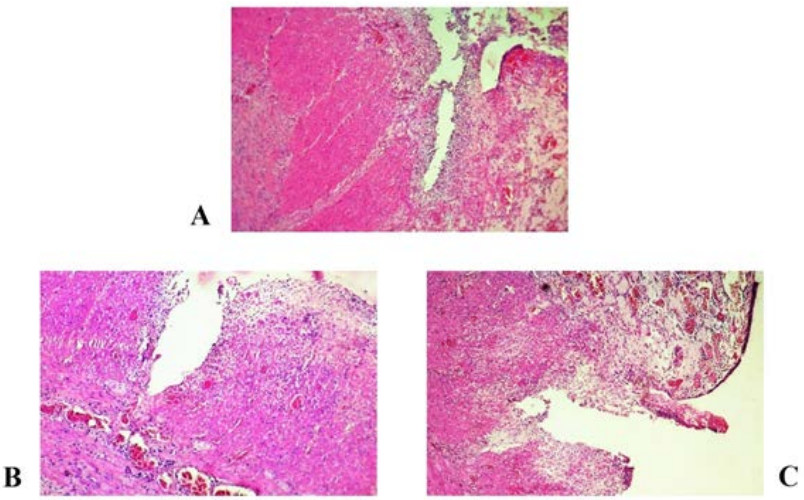

Figure 3 - Ulcers involving the mucosa, submucosa and breaching the muscularis propria. [(A) H\&E stain $4 \times$; (B) $H \& E$ stain $4 \times$; (C) $H \& E$ stain $4 \times]$ 


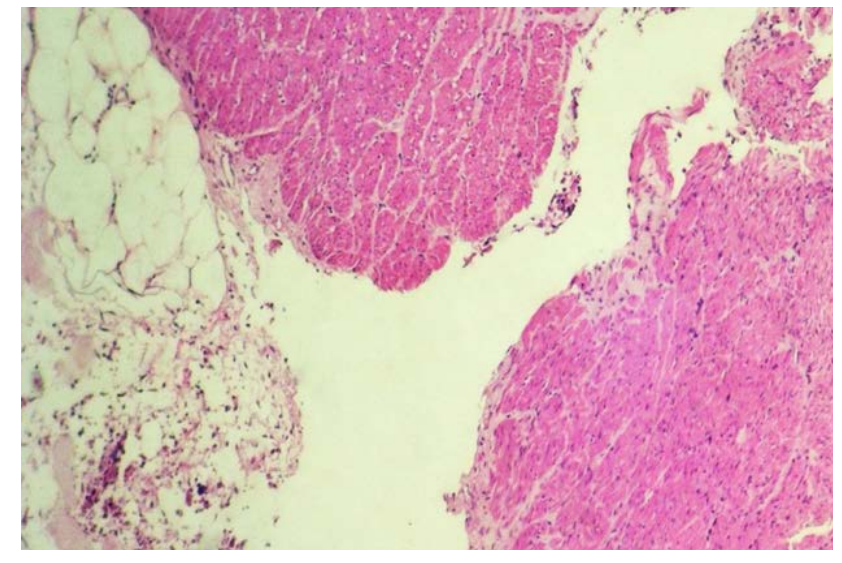

Figure 4 - Ulcer involving the entire intestinal wall, including the serosa. [H\&E stain 10×]

\section{Discussion}

The prevalence of Crohn's disease has been difficult to estimate because of the errors regarding the distinction between large intestinal Crohn's disease and ulcerative colitis [10]. Crohn's disease has a slightly higher incidence in females, and occurs at all ages [1]. It is considered an uncommon disease, with an estimated incidence of 5 to 20 cases per 100.000 population annually [1]. There are two peaks of incidence, one at 20 to 30 years of age, and the other in the $60-70$ age group $[1,10]$. Familial association is found in approximately $10 \%$ of the cases [1]. The apparent debut of Crohn's disease for our patient was, according to the questioning, at the age of 62 years.

The etiology of Crohn's disease remains completely unknown, although several theories have been issued, such as the involvement of genetic factors, environmental factors (including diet), and infective agents [10].

Concerning clinical features, the initial presentation may be indolent or may be acute and severe [1]. The symptoms are variable but often include cramping pain, typically localized to the right lower quadrant, nonbloody diarrhea, as well as fever, malaise, and anorexia [1]. Hemorrhage and hematochezia are uncommon, but patients may present iron deficiency anemia due to occult gastrointestinal bleeding [1]. Extraintestinal manifestations, when present, involve inflammatory changes in joints, eyes, liver, and skin [1].

Because resection is typically performed only in patients who have severe complications, the gross pathologic findings will be often significant [1]. The subserosal fat is often firm and contracted over the areas of involvement [1]. Longitudinal opening of the bowel may reveal areas with normal bowel wall thickness and other areas of the bowel that are firm, thickened and pipelike [1]. Interloop adhesions occur [1]. The mucosa may show a variety of changes, including aphthous erosions, longitudinal "rake" ulcers, cobblestoning, polyps, fissures, and fistulas [1].The process is usually multifocal, often with rectal sparing, but may become confluent [1]. When confluent, the firm pipelike nature of the bowel may help to distinguish Crohn's disease from ulcerative colitis, where fibrosis is unusual [1].

The most characteristic microscopic features of the disease are its multifocal involvement, focal ulceration and transmural inflammation in the form of lymphoid aggregates and granulomas [10]. It is important to know that granulomas may be absent or poorly formed $[1,11]$. When found, ulcers are typically longitudinally oriented, separated by histologically normal edematous mucosa [1]. When granulomas and fissuring ulcerations are absent, the most prevalent and diagnostically useful feature is transmural inflammation in the form of multiple lymphoid aggregates, scattered throughout the bowel wall but particularly obvious in the submucosa and subserosa [10].

No specific tests for Crohn's colitis are available. In cases in which distinguishing between ulcerative colitis and Crohn's colitis is difficult, performing both ASCA(anti-Saccharomyces cerevisiae antibody) and P-ANCA tests may help in the differential diagnosis because P-ANCA test results are positive in approximately $70 \%$ of patients with ulcerative colitis and in $10 \%$ to $30 \%$ of patients with Crohn's disease [1].The overlap of results between Crohn's disease and ulcerative colitis impose further research, thus underlining the importance of a histopathological confirmation [1].Fulminant Crohn's colitis can be 
difficult or impossible to distinguish from ulcerative colitis in a mucosal biopsy specimen; furthermore, in fulminant (toxic) ulcerative colitis, inflammation may become transmural [1]. The effects of treatment can also cause diagnostic difficulty because treated ulcerative colitis may show areas of involved and uninvolved mucosa and variable degrees of activity [1].

Crohn's colitis must be also distinguished from infectious colitis and medication-associated colitis; histologically, the erosions of Crohn's colitis are associated with a chronic inflammatory infiltrate, while in infectious colitis is characterized by a predominance of neutrophils rather than lymphocytes and plasma cells [1]. Medication-induced erosions usually have negligible acute inflammation, and typically only a few neutrophils are seen in the area of erosion [1]. Another important differential diagnosis of Crohn's disease is tuberculosis. Histological features favoring a diagnosis of tuberculosis include the number, size and coalescence of granulomas and the presence of caseating necrosis [12]. Transmural inflammation in the form of lymphoid aggregates, small granulomas, focal inflammation and poorly formed granulomas favor Crohn's disease [12].

No cure for Crohn's disease was found. Treatment focuses on inducing and maintaining remission, minimizing side effects, and improving the quality of life [1]. Standard therapy includes antiinflammatory drugs, mainly 5-ASA (5-aminosalicylic acid) compounds as sulfasalazine and other salicylates, and steroids; infliximab, a monoclonal antibody against tumor necrosis factor- $\alpha$, has been found to be helpful in treating acute episodes of Crohn's colitis, particularly fistulas and other complications [1]. A correct diagnosis in an early stage of the disease's evolution is ideal not only because it implies avoiding local and systemic complications, but also because of the increased risk for adenocarcinoma in patients with Crohn's disease [1]. Multiple synchronous or metachronous adenocarcinomas may occur and involve the large and small intestines [1]. Overall mortality for adenocarcinomas arising in Crohn's disease is high, approximately $80 \%$, mainly because of the similarities between symptoms of Crohn's disease relapse and symptoms of developing cancer, which may lead to a delayed diagnosis of the adenocarcinoma [1].

\section{Conclusion}

Although considered by many an uncommon entity which affects mainly young adults, it is important to know that Crohn's disease may debut at any age. The symptoms for which patients may require medical attention can mimic a wide variety of disorders whose treatments greatly differ. Establishing an appropriate treatment in order to avoid short and long term complications, which may be life-threatening to the patient, depends mainly on distinguishing between other inflammatory disorders of the digestive tract and Crohn's disease. A histopathologically confirmed diagnosis becomes greatly necessary also because of the increased risk of adenocarcinoma in patients with Crohn's disease.

\section{References}

1. Iacobuzio-Donahue, C.A. \& Montgomery, E.A. (2012). Idiopathic inflammatory bowel disease. In J.R. Goldblum (series editor), Gastrointestinal and Liver Pathology. USA: Elsevier Saunders

2. Dalziel, T.K. (1913). Chronic interstitial enteritis. Br Med J, ii, 1068

3. Lockhart-Mummery, H.E. \& Morson, B.C. (1960). Crohn's disease (regional enteritis) of the large intestine and its distinction from ulcerative colitis. Gut. 1, 87

4. Morson, B.C. \& Lockhart-Mummery, H.E. (1959). Anal lesions in Crohn's disease. Lancet. ii, 1122

5. Fielding, J.F., Toye, D.K., Beton, D.K. \& Cooke, 
W.T. (1970). Crohn's disease of the stomach and duodenum. Gut. 11, 1001

6. Basu, M.K., Asquith, P., Thompson, R.A. \& Cooke, W.T. (1974). Proceedings: Oral lesions in patients with Crohn's disease. Gut. 15, 346

7. Huchzermeyer, H., Paul, F., Seifert, E., Frohlich, H. \& Rasmussen, C.W. (1977). Endoscopic results in five patients with Crohn's disease of the esophagus. Endoscopy. 8, 75

8. Rankin, G.B., Watts, H.D., Melnyk, C.S.\& Kelley, M.L. Jr. (1979). National Cooperative Crohn's Disease Study: extraintestinal manifestations of perianal complications. Gastroenterology. 77, 914

9. Greenstein, A.J., Sachar, D.B., Smith, H., Janowitz, H.D. \& Aufses, A.H. Jr. (1980). Patterns of neoplasia in Crohn's disease and ulcerative colitis. Cancer. 46, 403
10. Day, D.W., Jass, J.R., Price, A.B., Shepherd, N.A., Sloan, J.M., Talbot, I.C., Warren, B.F. \& Williams, G.T. (2003). Inflammatory disorders of the small intestine (pp. 288-298); Inflammatory disorders of the large intestine (pp. 504-511). In: Morson and Dawson Gastrointestinal Pathology. Blackwell Science, Blackwell Publishing Company.

11. Heimann, T.M., Miller, F., Martinelli, G., Szporn, A., Greenstein, A.J. \& Aufses, A.H. Jr. (1988). Correlation of presence of granulomas with clinical and immunological variables in Crohn's disease. Arch Surg. 123, 46

12. Pulimood, A.B., Ramakrishna, B.S. \& Kurian, G. et al. (1999). Endoscopic mucosal biopsies are useful in distinguishing granulomatous colitis due to Crohn's disease from tuberculosis. Gut. 45,537 